\title{
Micro/mini-dystrophines et dystrophie musculaire de Duchenne : entre espoirs et défis
}

\author{
Emmanuelle Lagrue, Claude Cancès, Juliette Ropars
}

Les nombreux travaux précliniques de thérapie génique (TG) mis en œuvre afin de modifier l'histoire naturelle de la dystrophie musculaire de Duchenne (DMD), ont aujourd'hui abouti à la mise en place d'essais cliniques évaluant la sécurité et l'efficacité de l'administration de mini- ou micro-dystrophines chez l'enfant, et à terme peut-être chez l'adulte. Nous reprendrons dans cet article le principe général de la TG, les modèles animaux étudiés, les essais cliniques avec mini- ou micro-dystrophine actuellement en cours, et enfin les limites et effets indésirables possibles de ce type de thérapeutique innovante.

\section{Principe général de la thérapie génique}

Le principe de la thérapie génique dans le traitement des maladies monogéniques est d'intégrer, de manière complète ou partielle, le gène responsable d'une pathologie donnée (on parle de "transgène ") dans un vecteur le plus souvent viral (lentivirus, gamma-rétrovirus, ou virus adéno-associé [AAV]), l'expression du gène étant régulée par un promoteur.

Dans la TG ex vivo, laquelle associe thérapie cellulaire et thérapie génique proprement dites, des cellules du patient sont prélevées, exposées au vecteur viral, puis réinjectées au patient, alors que dans les TG in vivo, le vecteur viral est directement administré par voie sanguine générale (administration systémique) ou loco-régionale. Travailler ex vivo permet de mieux contrôler les étapes, d'utiliser moins de vecteurs et d'éviter les effets dits "off target " (atteinte d'organes non ciblés du fait de la dispersion potentielle du traitement). Cette solution est la plus souvent utilisée pour le traitement des maladies hématologiques, car il est possible de prélever les cellules à corriger par une simple prise de sang. La TG in vivo est quant à elle utilisée actuellement pour des pathologies musculaires, respiratoires, oculaires, ou neurologiques. Dans le cas de la DMD, l'administration in vivo permet ainsi potentiellement le traitement simultané des muscles squelettiques, du muscle cardiaque, et du diaphragme [1]. Un élément critique dans le processus de TG est le vecteur lui-même. Les vecteurs lentiviraux et les
AAV ont progressivement remplacé les vecteurs adénoviraux et gamma-rétroviraux initialement utilisés lesquels exposaient à d'importantes réactions immunitaires. Les AAV recombinants sont dits non intégratifs, c'est-à-dire que le transgène pénètre jusqu'au noyau cellulaire cible mais sans s'intégrer dans le génome (il persiste donc dans le noyau sous forme d'épisome). Ceci limite le risque de survenue d'éventuels cancers post-TG liés à l'intégration accidentelle du transgène à proximité d'oncogènes. A contrario, on risque de voir apparaitre une "dilution " du transgène au cours des mitoses du fait de son caractère épisomal.

L'utilisation des AAV est également limitée par le fait que les patients peuvent avoir été en contact préalable avec un AAV "sauvage " d'un sérotype donné, avec comme conséquence la production d'anticorps neutralisants anti-AAV. Cette immunisation acquise rend par définition toute tentative de TG inopérante. La prévalence relative des différents anticorps anti-AAV dans la population des garçons atteints de DMD peut donc impacter le choix du vecteur parmi les trois les plus utilisés qui sont l'AAV9, l'AAV8 et le rh74, ce dernier étant sérotypiquement proche de l'AAV8 [2].

L'autre limitation du recours aux AAV réside dans la taille extrême du gène $D M D$ codant la dystrophine. Ce gène dont la partie codante (11,2 kilobases) pouvait être contenue en entier dans un adénovirus ne peut l'être dans un AAV dont la capacité de stockage est bien moindre (4,7 kilobases environ). Le gène $D M D$ est divisé en quatre domaines (N-terminal, central/rod, riche en cystéine et C-terminal). Le domaine central est sous-divisé en 24 répétitions spectrine et 4 charnières. L'utilisation de vecteurs non-viraux de type plasmide pourrait lever l'obstacle de la taille du gène DMD à l'avenir. Dans l'attente, il s'avère donc nécessaire de bien "choisir" les domaines du gène $D M D$ capables de générer une mini- ou une micro-dystrophine (Figure 1). Le terme de mini-dystrophine renvoie aux montages contenant des séquences codant un domaine C-terminal complet aboutissant à un transgène de 6 à $8 \mathrm{~kb}$. L'administration de ces mini-dystrophines requiert l'utilisation concomitante de deux AAVs. 
A

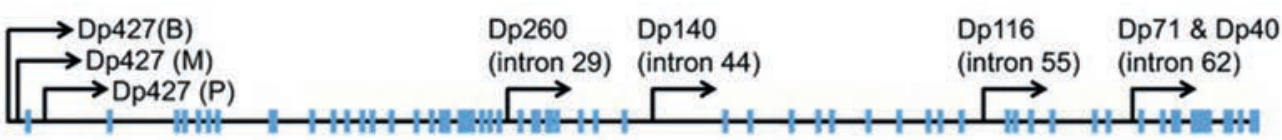

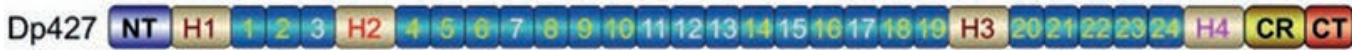

Dp260 1011121374151017 1019 H3 2021222924 H4 CR CT

Dp140 10 H3 2024e2ese4 H4 CR CT

Dp116 222024 H4 CR CT

Dp71 CRCT

Dp40 CR

B Full-length dystrophin AB1 $\quad$ AB2 nNOS

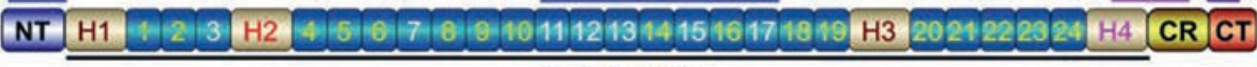

Rod domain
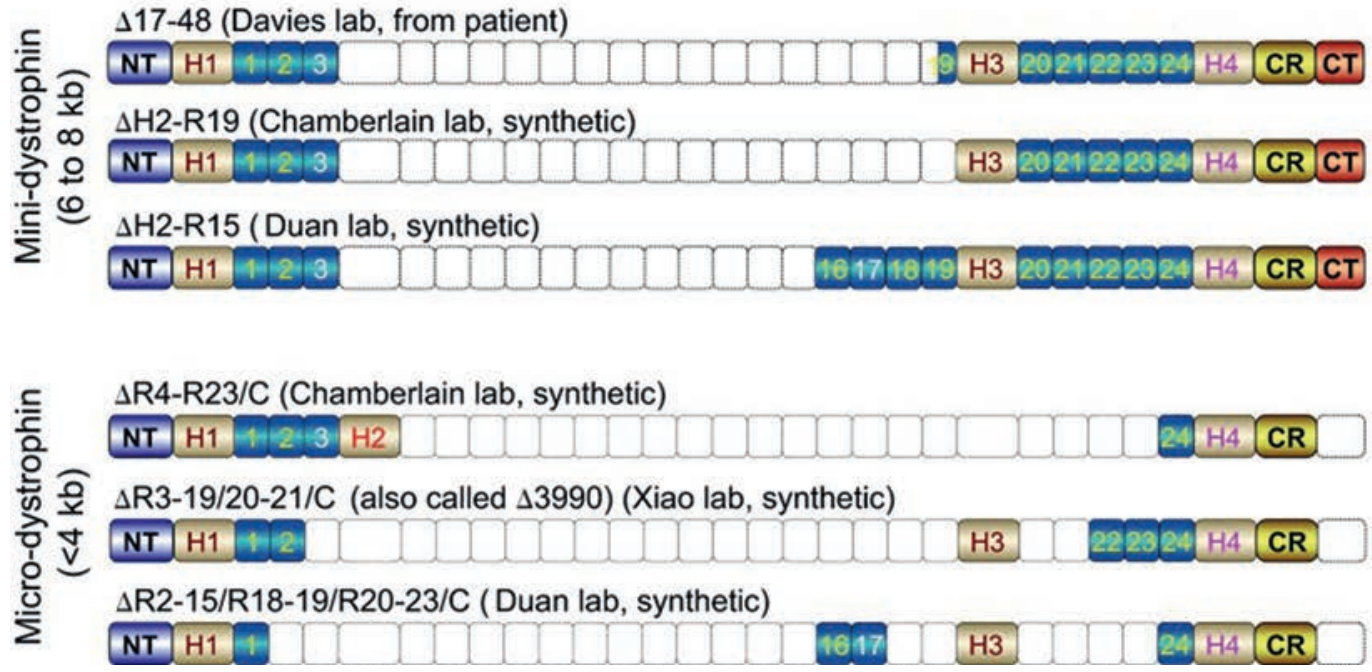

Figure 1

Structure des micro et mini-dystrophines (d'après [3]).

A. Isoformes naturelles. Les transcrits Dp427 ont 3 isoformes dans le cerveau Dp427(B), le muscle Dp427(M) et les cellules de Purkinje Dp427(P). Des isoformes plus courtes sont produites dans la rétine (Dp260), le cerveau et le rein (Dp140), les cellules de Schwann (Dp116).

B. Structure des mini- et micro-dystrophines.

Les transgènes produisant les micro-dystrophines ne mesurent quant à eux que 3,5 à $4 \mathrm{~kb}$, et ne codent pas un domaine $\mathrm{C}$-terminal complet, ce qui aboutit à la production d'une protéine mesurant environ un tiers de la taille normale [4]. Elles peuvent donc être administrées avec un AAV unique.

Le choix des domaines du gène $D M D$ retenus dans la constitution de ces mini-/micro-dystrophines est basé sur leur rôle physiologique respectif. Les domaines en charge de l'interaction avec le cytosquelette et de la production de radicaux libres dans le sarcolemme sont nécessaires à l'obtention d'une récupération fonctionnelle. L'intégration d'un nombre pair de répétitions spectrine pourrait améliorer le fonctionnement du domaine central [5], mais l'augmentation à 5 domaines majore la taille du 
transgène de manière significative. De même, l'intérêt d'intégrer ou pas la charnière 3 n'a pas encore été tranché, alors qu'il a été clairement démontré qu'il valait mieux exclure la charnière 2 [6]. Au-delà du vecteur et de la composition du transgène, le choix du promoteur est également un critère majeur d'efficacité et de sécurité. C'est ainsi qu'un promoteur spécifique du muscle (de type CK8, MHCK7, miniMCK, ou SPc5-12) est systématiquement choisi pour limiter les possibles effets dits " off-target" [7].

\section{Modèles animaux}

Avant leur passage chez l'homme, les mini-/microdystrophines ont d'abord été évaluées à l'aide de modèles animaux. Un transgène de type micro-dystrophine a ainsi montré une amélioration de la force musculaire et de la fonction cardiaque chez la souris et le chien, les sérotypes utilisés étant les AAV6, 8 et 9. En 2017, une avancée majeure est obtenue lorsqu'une équipe française démontre une efficacité tangible chez le golden retriever, le modèle chien de la $\mathrm{DMD}$, après administration locorégionale ou systémique, médiée par un vecteur $\mathrm{rAAV} 2 / 8$, d'une micro-dystrophine canine. Cette expérimentation réalisée sur douze chiens a confirmé une restauration de l'expression de la micro-dystrophine et s'est accompagné d'une amélioration des éléments dystrophiques observés sur biopsie musculaire. De surcroît, cet effet s'était maintenu pendant plus de deux ans, sans toxicité ni conséquences immunitaires indésirables [8].

Des expérimentations complémentaires sont en cours pour tenter d'obtenir une meilleure expression de la micro-dystrophine, notamment en condition de modulation immunitaire [9]. Une amélioration du transgène est également visée. Il semble que les micro-dystrophines comportant des domaines de type spectrine R16-R17 (domaines impliqués dans la liaison avec le régulateur du métabolisme cellulaire via l'oxyde nitrique synthase), pourraient avoir des avantages thérapeutiques supplémentaires [10].

\section{Essais thérapeutiques en cours}

La preuves d'innocuité et d'efficacité obtenues dans des modèles animaux ont permis le passage à l'homme et donc la mise en route de plusieurs essais cliniques. De nombreux essais à base de mini-/microdystrophines ont été menés et/ou sont encore en cours [11] respectivement par les laboratoires Pfizer (PF-06939926, NCT03362502), SareptaTherapeutics (NCT03375164, NCT03769116), Solid Biosciences (NCT03368742), avec utilisation de sérotypes AAV9 ou AAVrh74 à des doses élevées de vecteurs (allant de $1 \times 10^{14}$ à $3 \times 10^{14}$ copies de génome viral/kg ou vg/kg) [12] et enfin Généthon. L'essai récent mené par les équipes de Généthon utilise une dose un log plus faible $\left(10^{13}\right)$. Les principales caractéristiques de ces essais sont résumées dans le Tableau I.

Concernant l'essai conduit par Sarepta, le choix retenu pour le vecteur AAV a été celui du sérotype rh74. Ce sérotype est peu rencontré à l'état naturel chez les patients atteints de DMD. Il induirait une réponse immunitaire moindre et a prouvé son innocuité lors des études précliniques à doses croissantes [10]. La dose minimale efficace validée dans le modèle de souris $m d x$ a été de $2 \times 10^{14} \mathrm{vg} / \mathrm{kg}$. La première étude de phase I chez l'homme a consisté en une injection unique chez quatre patients à cette dose d'AAVrh74-MHCK7-coDR4-R23DCT (SRP9001). Les premiers résultats sont encourageants en termes de sécurité, avec une toxicité hépatique transitoire et résolutive sous glucocorticoïdes, ainsi qu'une réaction immunitaire indésirable modérée. La micro-dystrophine était fortement exprimée (81,2\% des fibres musculaires) au niveau du biceps brachial [13]. Bien qu'une amélioration clinique significative de 2,2 à 7 points sur l'échelle fonctionnelle North Star Ambulatory Assessment (NSAA) ait été observée, tout comme une baisse du taux sérique des CPK, l'efficacité de ce transgène reste à démontrer auprès d'un échantillon plus large de patients, sur une période d'évaluation prolongée, et en comparaison à une cohorte de patients non traités (histoire naturelle). Une deuxième étude de phase I (NCT04626674) est en cours auprès de 20 patients, avec pour objectif d'évaluer l'expression de la microdystrophine, douze semaines après l'injection. Un essai de phase II randomisé, contrôlé versus placebo (SRP-9001, Sarepta) est également en cours aux États-Unis, auprès de 41 garçons âgés de 4 à 7 ans (NCT03769116). Les résultats à 48 semaines n'ont pas permis de détecter de différence significative entre les deux groupes, malgré une ré-expression de la dystrophine sur la biopsie musculaire. Les garçons traités par TG ont ainsi eu une amélioration moyenne de 1,7 point de l'échelle fonctionnelle NSAA contre 0,9 point dans le groupe placebo. Le promoteur de l'étude a mis en avant, pour expliquer ce résultat décevant, un biais de randomisation avec une sévérité clinique plus marquée à l'inclusion dans le groupe traité. Une étude pré-spécifiée limitée aux enfants de 4 et 5 ans a par ailleurs détecté une différence significative (+4,3 de l'échelle NSAA dans le groupe traité contre $+1,9$ dans le groupe placebo), qui amène le laboratoire Sarepta à réfléchir à une poursuite éventuelle de l'étude avec adaptation du protocole. Des sites cliniques seront potentiellement ouverts en Europe en 2021 dans ce contexte. 
Tableau I

Principales

caractéristiques des essais thérapeutiques en cours.

\begin{tabular}{|c|c|c|c|c|c|c|}
\hline Nom de l'essai & Molécule & Promoteur & Phase & $\begin{array}{l}\text { Population } \\
\text { DMD éligible }\end{array}$ & Localisation & $\begin{array}{l}\text { Période } \\
\text { (début-fin } \\
\text { estimée) }\end{array}$ \\
\hline $\begin{array}{l}\text { SRP-9001 } \\
\text { NCT03375164 }\end{array}$ & $\begin{array}{l}\text { AAVrh74.MCK.micro-dystro- } \\
\text { phine }\end{array}$ & $\begin{array}{l}\text { Sarepta- } \\
\text { Therapeutics }\end{array}$ & I/II & $\begin{array}{l}\mathrm{N}=4 \\
\text { (3 mois-7 ans) }\end{array}$ & États-Unis & $2018-2023$ \\
\hline $\begin{array}{l}\text { SRP-9001 } \\
\text { NCT03769116 }\end{array}$ & $\begin{array}{l}\text { AAVrh74.MCK.micro-dystro- } \\
\text { phine }\end{array}$ & $\begin{array}{l}\text { Sarepta- } \\
\text { Therapeutics }\end{array}$ & II & $\begin{array}{l}N=41 \\
\text { (4 à } 7 \text { ans) }\end{array}$ & États-Unis & $2018-2026$ \\
\hline $\begin{array}{l}\text { PF-06939926 } \\
\text { NCT03362502 }\end{array}$ & AAV9.CK.micro-dystrophine & Pfizer & $\mathrm{Ib}$ & $\begin{array}{l}\mathrm{N}=30 \\
\text { (4 à } 12 \text { ans) }\end{array}$ & États-Unis & $2018-2026$ \\
\hline $\begin{array}{l}\text { PF-06939926 } \\
\text { NCT04281485 }\end{array}$ & AAV9.CK.micro-dystrophine & Pfizer & III & $\begin{array}{l}\mathrm{N}=99 \\
\text { (5 à } 12 \text { ans) }\end{array}$ & $\begin{array}{l}\text { Canada, } \\
\text { Corée, } \\
\text { Espagne, } \\
\text { USA, France, } \\
\text { Israël, Italie, } \\
\text { Japon, Russie }\end{array}$ & $2020-2027$ \\
\hline $\begin{array}{l}\text { SGT-001 } \\
\text { NCT03368742 }\end{array}$ & AAV9.CK8.micro-dystrophine & $\begin{array}{l}\text { Solid } \\
\text { Biosciences }\end{array}$ & $\mathrm{I} / \mathrm{II}$ & $\begin{array}{l}\mathrm{N}=16 \\
\text { (4 à } 17 \text { ans) }\end{array}$ & États-Unis & $2017-2027$ \\
\hline GNT-016 & AAV8.micro-dystrophine & $\begin{array}{l}\text { Généthon } \\
\text { Sarepta- } \\
\text { Therapeutics }\end{array}$ & I/II/III & $\begin{array}{l}\mathrm{N}=9 \\
\text { (escalade de } \\
\text { dose) puis } 42 \\
\text { (phase } \\
\text { pivotale) } \\
\text { (5-10 ans) }\end{array}$ & $\begin{array}{l}\text { États-Unis, } \\
\text { France, Israël, } \\
\text { Royaume-Uni }\end{array}$ & $2020-2028$ \\
\hline
\end{tabular}

Le laboratoire Pfizer mène actuellement deux essais de phase I et de phase III. L'essai de phase I a pour but de déterminer la dose optimale, l'innocuité et la tolérance d'une perfusion intraveineuse de PF06939926 (une mini-dystrophine médiée par AAV9 et désormais dénommée fordadistrogene movaparvovec) chez de 30 patients DMD ambulatoires et non ambulatoires (NCT03362502). Il est à noter que deux patients injectés ont présenté une toxicité rénale de type syndrome hémolytique et urémique atypique [14], appelée aussi microangiopathie thrombotique aiguë (ou MAT). CIFFREO, un essai de phase III multicentrique (55 centres estimés, dans 15 pays, 2 centres potentiels en France), randomisé, en double aveugle, contre placebo, est en place depuis 6 mois pour évaluer l'efficacité et l'innocuité du même vecteur PF-06939926. Au total, quatre-vingt-dix-neuf garçons atteints de $\mathrm{DMD}$, âgés entre 4 et 7 ans (inclusif) et capables de marcher, seront recrutés. Deux tiers recevront la TG à la semaine 1 , et un tiers recevra initialement le placebo, puis la TG à la semaine 52. L'objectif principal de l'étude est la modification de la NSAA à 52 semaines par rapport aux chiffres à l'inclusion. Les patients seront suivis pendant cinq ans après l'administration du vecteur.

Un troisième essai, de phase I/II, utilisant une microdystrophine véhiculée par un vecteur AAV9 (SGT001) élaborée par le laboratoire Solid Bioscience, a été interrompu durant plusieurs mois pour cause de sécurité (NCT03368742, essai IGNITE DMD). Une activation du complément, telle qu'observée dans l'essai de Pfizer, est en effet survenue chez 2 patients. L'agence américaine du médicament (FDA) a autorisé la reprise de l'essai en octobre 2020, suite à la démonstration par le laboratoire d'une réduction de la charge virale totale par élimination de la majorité des capsides vides.

Une quatrième série d'essais, comprenant une association de phase I/II puis phase III, vient de débuter, initiée par Généthon (GNT), en collaboration avec Sarepta, avec un transgène micro-dystrophine médié par un vecteur AAV8. Une histoire naturelle (étude GNT014) a été mise préalablement en place durant 3 à 36 mois, suivie, pour les garçons répondant aux critères d'inclusion, d'une phase d'essai thérapeutique. Cet essai (étude GNT016) comporte en premier lieu une phase I/II d'escalade de dose destinée à déterminer la dose optimale. Suivra ensuite une phase III, multicentrique, randomisée contre placebo, avec inclusion de 42 patients (NCT03882827) dont 21 recevront la TG à la semaine 1 , et 21 à la semaine 52 . La première injection a eu lieu en avril 2021. Sept centres seront ouverts en France, au Royaume-Uni, aux États-Unis et en Israël.

\section{Limitations et effets indésirables potentiels de la TG dans la DMD}

Le transfert de mini-/micro-dystrophines est arrivé au stade de la recherche clinique et suscite légitimement de grands espoirs. Cet enthousiasme ne doit néanmoins pas faire oublier les trois limitations majeures de cette technique. Comme évoqué 
antérieurement, la capacité d'encapsidation des AAV ne permet pas d'introduire un gène $D M D$ complet et permet donc difficilement d'envisager une stabilisation complète du phénotype. Deuxièmement, les patients déjà immunisés contre des AAV donnés ne sont pas éligibles à ce type de thérapie. L'apparition d'anticorps suite à l'administration de la TG ne permettra pas chez les garçons traités une "ré-administration". Cette "ré-administration " pourrait pourtant s'avérer nécessaire en cas d'épuisement de l'effet du transgène thérapeutique au-delà d'un délai encore difficile à prédire (8 à 10 ans?). Plusieurs équipes de recherche travaillent activement sur le sujet pour contourner cet obstacle conceptuel.

Enfin, la TG expose à un risque de réaction immunitaire pathologique, consistant d'une part en une activation du complément et d'autre part en une réponse cellulaire, toutes deux ayant parfois des conséquences dramatiques.

\section{Activation du complément}

Le complément est une cascade protéolytique constituée d'une trentaine de protéines solubles ou membranaires responsable de la détection et la destruction des pathogènes. Le complément comprend trois voies (voie classique, voie liée à la lectine, et voie alterne) qui convergent vers le clivage du composé C3. Une activation du complément peut donc survenir après infection par l'AAV, comme après toute infection virale.

L'activation du complément amène dans les stades précoces à la libération des anaphylatoxines C3a et C5a, et de cytokines (interleukine 1 et TNF). Différents composants du complément ayant la capacité de se fixer sur les récepteurs plaquettaires et/ou interagissant avec des facteurs de coagulation, une activation des plaquettes et de la coagulation se déclenche secondairement, avec effet d'auto entretien d'un système activé sur l'autre, créant une boucle d'amplification délétère [15].

Ceci engendre une microangiopathie thrombotique, c'est-à-dire un tableau associant une thrombopénie non auto-immune, une anémie hémolytique, et une atteinte d'organe [16]. Si l'activation du complément est généralisée, une défaillance touchant plusieurs organes et/ou une coagulation intravasculaire disséminée peuvent survenir. L'atteinte sera néanmoins le plus souvent limitée à un organe, préférentiellement le rein, avec un tableau de syndrome hémolytique et urémique atypique (aSHU) [17]. La survenue d'aSHU a été rapportée récemment chez trois enfants ayant reçu une thérapie génique par onasemnogene abeparvovec (Zolgensma ${ }^{\circledR}$ ) dans le cadre du traitement de l'amyotrophie spinale infantile de type 1 [18]. Le même type de complication est survenu, tel qu'évoqué antérieurement, dans l'essai micro-dystrophine mené par le laboratoire Solid. La survenue d'un aSHU reste imprévisible et peut avoir des conséquences dramatiques même si un traitement à base d'éculizumab pourrait permettre d'enrayer l'emballement immunitaire. L'effet indésirable semble être dose-dépendant [12]. Ceci pourrait limiter la réalisation d'une TG chez les patients au-dessus d'un certain poids, et Solid a décidé de restreindre l'administration aux garçons pesant moins de $18 \mathrm{~kg}$ une fois la reprise de l'essai autorisée par la FDA.

\section{Réponse cellulaire}

Au-delà de l'activation du complément, l'introduction d'un AAV dans l'organisme peut déclencher une réaction immune cellulaire [16]. Ceci a été bien documenté dans des essais de TG pour l'hémophilie [20] et pourrait expliquer l'échec du premier essai clinique sur la mini-dystrophine durant lequel les patients traités avaient produit des cellules T spécifiques contre la mini-dystrophine alors que la protéine elle-même n'était pas exprimée [21].

La corticothérapie prescrite dans les semaines suivant la TG a pour objectif de maitriser cette immunotoxicité, et permet également de diminuer la réaction hépatique liée au tropisme préférentiel pour le foie des vecteurs viraux utilisés.

\section{En conclusion}

La thérapie génique par mini/micro-dystrophine est une des approches thérapeutiques les plus prometteuses chez les patients présentant une DMD. Les essais thérapeutiques en cours et à venir permettront d'établir l'efficacité de la transduction obtenue, de déterminer la meilleure composition possible du transgène, de prouver l'impact clinique de la protéine produite, et d'évaluer la sécurité de ces procédures.

Micro/mini-dystrophins and Duchenne muscular dystrophy: between hopes and challenges

\section{LIENS D'INTÉRÊT}

Les auteurs déclarent n'avoir aucun lien d'intérêt concernant les données publiées dans cet article.

\section{RÉFÉRENCES}

1. Mendell JR, Al-Zaidy SA, Rodino-Klapac LR. Current clinical applications of in vivo gene therapy with AAVs. Mol Ther 2021; $29: 464-88$.

2. Louis Jeune V, Joergensen JA, Hajjar RJ, Weber T. Pre-existing anti-adeno-associated virus antibodies as a challenge in AAV gene therapy. Hum Gene Ther Methods 2013 ; 24 : 59-67.

3. McGreevy JW, Hakim CH, McIntosh MA, et al. Animal models of Duchenne muscular dystrophy: from basic mechanisms to gene therapy. Dis Model Mech $2015 ; 8$ : 195-213. 
4. Duan D Systemic AAV micro-dystrophin gene therapy for Duchenne muscular dystrophy. Mol Ther 2018 ; 26 : 2337-56. 5. Harper SQ, Hauser MA, DelloRusso C, et al. Modular flexibility of dystrophin: implications for gene therapy of Duchenne muscular dystrophy. Nat Med 2002 ; 8 : 253-61.

6. Banks GB, Judge LM, Allen JM, et al. The polyproline site in hinge 2 influences the functional capacity of truncated dystrophins. PLoS Genet $2010 ; 6$ : e1000958.

7. Shieh PB. Emerging strategies in the treatment of Duchenne muscular dystrophy. Neurotherapeutics $2018 ; 15$ : 840-8.

8. Le Guiner C, Servais L, Montus M, et al. Long-term microdystrophin gene therapy is effective in a canine model of Duchenne muscular dystrophy. Nat Commun 2017; $8: 16105$.

9. Hayashita-Kinoh H, Guillermo PH, Nitahara-Kasahara Y, et al. Long-term microdystrophin gene therapy is effective in a canine model of Duchenne muscular dystrophy. Mol Ther Methods Clin Dev 2020 ; 20 : 122-41

10. Buscara L, Gross DA, Daniele N. Of rAAV et men: from genetic neuromuscular disorder efficacy and toxicity preclinical studies to clinical trials and back. J Pers Med 2020 ; $10: 258$.

11. Hakim $\mathrm{CH}$, Wasala NB, Pan X, et al. A five-repeat microdystrophin gene ameliorated dystrophic phenotype in the severe dba/2j-mdx model of Duchenne muscular dystrophy. Mol Ther Methods Clin Dev 2017 ; 6 : 216-30.

12. Fortunato F, Rossi R, Falzarano MS, et al. Innovative therapeutic approaches for Duchenne muscular dystrophy. $J$ Clin $\mathrm{Med}$ $2021 ; 10: 820$
13. Mendell JR, Sahenk Z, Lehman K, et al. Assessment of systemic delivery of AAVrh74.MHCK7 micro-dystrophin in children with Duchenne muscular dystrophy: a nonrandomized controlled trial. JAMA Neurol 2020 ; 77 : 1129-31.

14. Pfizer's new phase $1 \mathrm{~b}$ results of gene therapy in ambulatory boys with Duchenne muscular dystrophy (DMD) support advancement into pivotal phase 3 studyPfizer - Communiqué de presse du 15 mai 2020.

15. Luo S, Hu D, Wang M, et al. Complement in hemolysis- and thrombosis-related diseases. Front Immunol 2020 ; 11: 1212.

16. Monteiro Pereira Palma L, Sridharan M, Sethi S. Complement in secondary thrombotic microangiopathy. Kidney Int Rep $2021 ; 6: 11-23$

17. Lemaire M, Noone D, Lapeyraque AL, et al. Inherited kidney complement diseases. Clin J Am Soc Nephrol 2021; 16 : $942-56$

18. Chand DH, Zaidman C, Arya K, et al. Thrombotic microangiopathy following onasemnogene abeparvovec for spinal muscular atrophy: a case series. J Pediatr 2021 ; 231 : 265-8.

19. Li C, Goudy K, Hirsch, et al. Cellular immune response to cryptic epitopes during therapeutic gene transfer. Proc Natl Acad Sci USA 2009; 106 : 10770-4

20. George LA. No CpGs for AAVs? Blood 2021 ; 137 : 721-3.

21. Mendell JR, Campbell K, Rodino-Klapac L, et al. Dystrophin immunity in Duchenne's muscular dystrophy. N Engl J Med 2010 ; 363 : 1429-37.

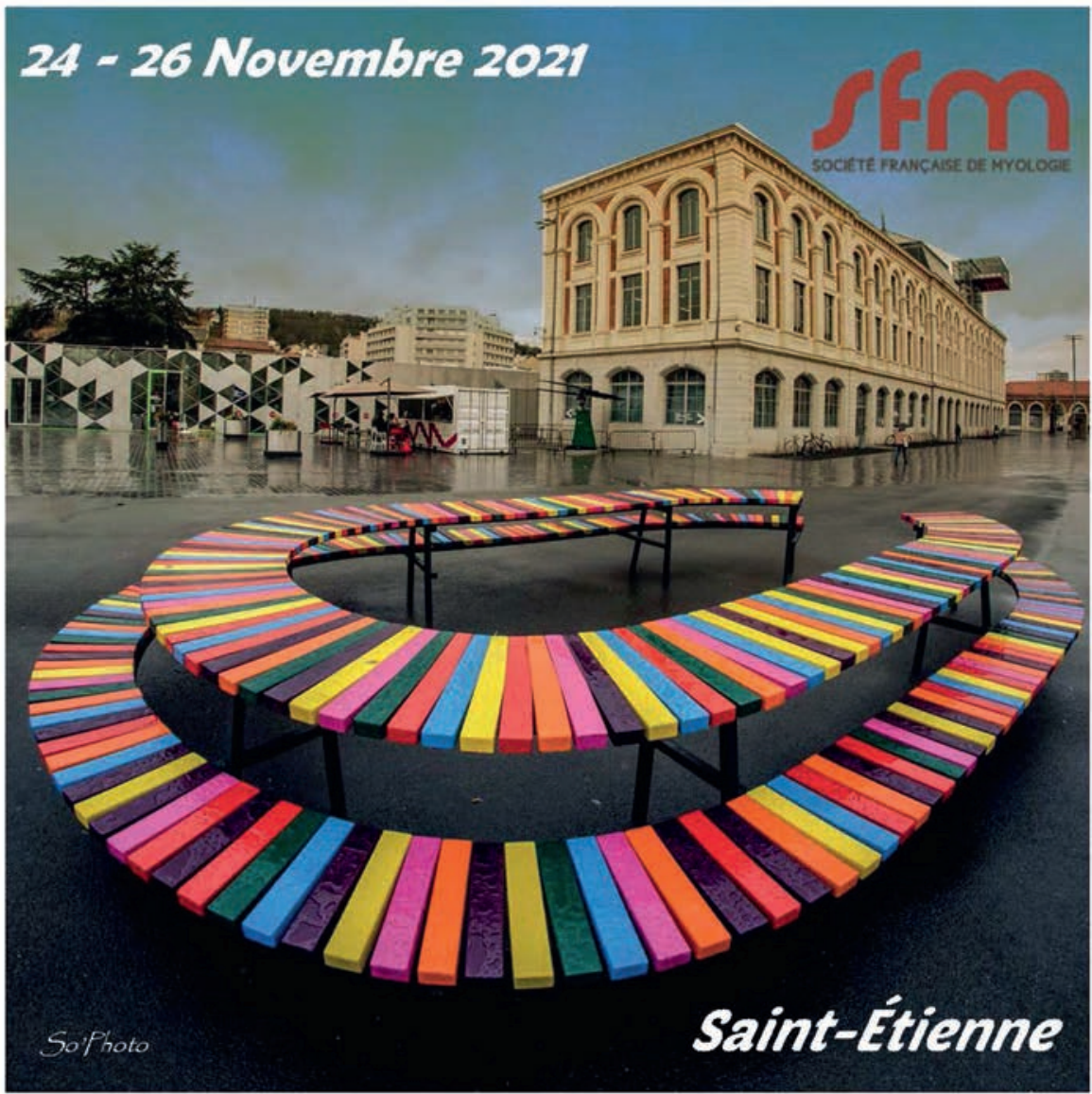

\section{$18^{\text {èmes }}$ Journées de la Société Française de Myologie}

www.jsfmyologie2021.com
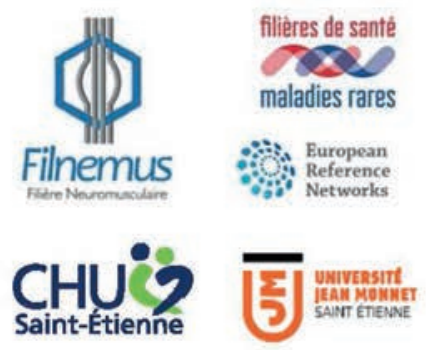

ActiFS

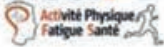

